
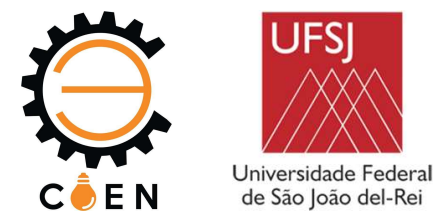

\title{
MÉTODO DA ENGENHARIA REVERSA PARA O DIMENSIONAMENTO DA GAIOLA HOMOCINÉTICA
}

Igor Damasceno Baeta (igor.d.mec3@gmail.com), Marcelo Esposte Purgato (marceloesposte@gmail.com), Alexandre Chaves de Melo (alexandrechavesdemelo@gmail.com), Marina Paiva Rodrigues (mapaivarodrigues@yahoo.com.br)

\footnotetext{
Universidade Federal de São João Del Rei (UFSJ), Curso de Engenharia Mecânica, Praça Frei Orlando-170, Centro, 36307-904, São João Del Rei, Minas Gerais, Brasil.

Universidade Federal de São João Del Rei (UFSJ), Departamento de Engenharia Mecânica (DEMEC), Praça Frei Orlando-170, Centro,36307-904, (32)3379-5879, São João Del Rei, Minas Gerais, Brasil.
}

RESUMO: O protótipo baja é um veículo "Off Road" produzido para competições nas quais é submetido a uma série de provas, de diferentes modalidades, que avaliam seu desempenho, entre elas: aceleração, velocidade e capacidade de vencer aclives. O sistema de Powertrain é extremamente importante na maioria dos equipamentos mecânicos. Para os automóveis é ainda mais importante, por ser responsável pela locomoção do veículo. A transmissão de torque e potência, produzida pelo motor até as rodas, é realizada por todos os componentes, em conjunto, ao mesmo tempo. O funcionamento da transmissão obedece a um rigoroso e preciso circuito mecânico, da geração de força até os pneus. Sendo assim, é de suma importância considerar a máxima eficiência e a vida de todos os componentes. A gaiola homocinética é um componente que faz parte do conjunto homocinético de transmissão. Ela possui seis canais de seção circular onde são encaixadas as esferas de aço. No interior da gaiola é acoplado o anel interno que se encaixa no semieixo e assegura que as esferas permaneçam dentro dos canais. Por fim, todos esses componentes são partes intrínsecas do sino que é ligado ao cubo de roda. Devido a decorrentes quebras da gaiola, apresentamos este artigo que tem como objetivo analisar a utilização da engenharia reversa para a fabricação de gaiolas homocinéticas utilizando o método de seleção dos materiais. A escolha do material mais adequado, de acordo com as exigências do sistema de transmissão do protótipo, torna as gaiolas mais resistentes.

PALAVRAS-CHAVE: Baja, Powertrain, conjunto homocinético, gaiola homocinética, Engenharia Reversa

\section{INTRODUÇÃO}

Algumas invenções tecnológicas surgiram de estudos que tinham como base outras, e se desenvolveram de forma independente do objetivo inicial do estudo, como foi o caso da empresa AMD (Advanced Micro Devices, Inc.), que conseguiu fazer uma cópia exata do processador Intel 8080 em 1975. Outro exemplo são as pesquisas de tecnologias antigas, com carência de documentação descritiva. Com a utilização da engenharia reversa é possível que a documentação seja elaborada e a tecnologia utilizada seja especificada e melhor estudada (HAUTSCH, 2009).

A engenharia reversa está se tornando uma importante área de pesquisa para o avanço de tecnologias existentes, pois permite que mesmo aquilo que é protegido por leis de propriedade intelectual seja estudado e melhorado. É um método de exame, que começa com a análise do objeto de estudo em sua implementação existente, detalhando sua composição química, construção, formatos de peças e todos os seus componentes, com o objetivo de que todo o seu funcionamento seja conhecido e passível de inovação e da adição de novas aplicações (SANTOS, 2016).

Para a realização desse processo passa-se, basicamente, por quatro etapas: a digitalização 3D (captura de dados); tratamento dos dados coletados; criação de modelo CAD e, quando necessário, prototipagem rápida (PONTICELLI e SUSKI, 2017). 
Este artigo apresenta um estudo em que foi utilizado o método da engenharia reversa através de medição 3D para o dimensionamento da gaiola homocinética de um veículo baja SAE. O mesmo foi realizado pela equipe Komiketo Baja na Universidade Federal de São João del-Rei (UFSJ). O processo de medição $3 \mathrm{D}$ foi necessário devido ao fato do conjunto homocinético utilizado ter saído de série, além de não possuir registro de sua procedência.

Para extrair a geometria da gaiola homocinética foi feita a digitalização do modelo que a equipe possuía com o intuito de obter uma sequência de pontos no espaço, visando sua fabricação. Este processo foi realizado pela empresa "SIGMA - Medições 3D", prestadora de serviços, por meio do método de digitalização por contato.

$\mathrm{O}$ uso de juntas homocinéticas em automóvel é fundamental por proporcionar, nos movimentos de tração e direção, vários movimentos em diversos ângulos. Na Figura 1 tem-se uma ponta de eixo, também chamada de sino, que é ligada ao cubo da roda. No seu interior existem seis canais ou pistas, de seção semicircular, onde são encaixadas seis esferas de aço. Em conjunto com um anel, chamada "gaiola", essas esferas controlam a posição longitudinal nos canais. Acoplado a esses componentes está um anel interno, que possui seis canais similares aos do sino e que fica ligado ao semieixo.

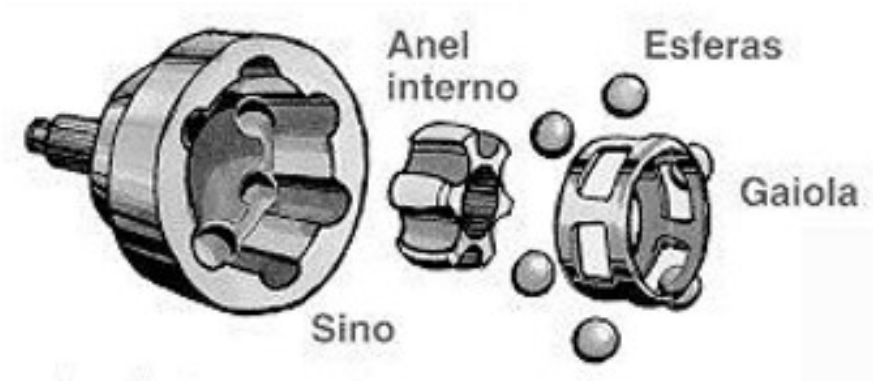

Figura 1. Vista explodida do conjunto homocinético identificando suas partes.

O movimento das esferas, somado aos esforços atuantes do movimento torçor vindo do redutor de engrenagens, da força de frenagem e do movimento da suspensão, que faz com que o conjunto do semieixo trabalhe em várias angulações, gera um desgaste nos componentes do conjunto homocinético.

A gaiola, por sua vez, é um componente que interliga o anel interno com as esferas, sofrendo esforços de contato de ambos para transmitir o movimento vindo do redutor de engrenagem para as rodas. Esses esforços causam desgaste e podem ser minimizados com a utilização de materiais com características apropriadas para esta finalidade.

Para isso, foi necessário observar algumas características da gaiola original, como sua dureza e microestrutura, a fim de determinar qual seria o melhor material e tratamentos térmicos que atendessem às propriedades originais.

\section{MATERIAIS E MÉTODOS}

A medição tridimensional da peça foi realizada pelo método de digitalização por contato, aquisição ponto a ponto, que consiste na varredura de dados coordenados, que são armazenados em um sistema de pontos. Para isso, um apalpador toca a peça em uma direção normal à mesma. Inicialmente, são adquiridas as coordenadas do ponto central da peça e em seguida os demais, com 


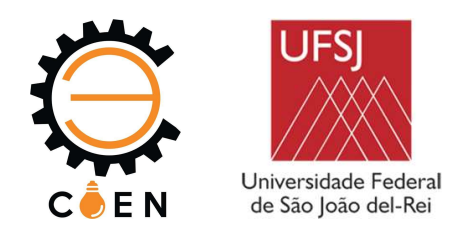

a correção do raio do sensor esférico (PILLON, 2015). Esse processo foi patrocinado pela empresa SIGMA - Medições 3D, localizada em São Paulo - SP. O desenho obtido pela medição pode ser visto na Figura 2.

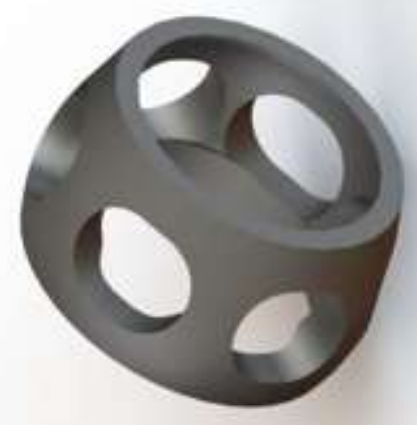

Figura 2. Desenho da gaiola homocinética obtido com a medição tridimensional.

Os processos de medição de dureza, ensaios metalográficos, microscopia óptica e de microscopia eletrônica de varredura foram realizados nos laboratórios do Departamento de Engenharia Mecânica (DEMEC) na Universidade Federal de São João del - Rei (UFSJ). O ensaio de microdureza foi feito com um durômetro Rockwell.

Devido a procedência desconhecida da peça original e, consequentemente, o não conhecimento da composição química do componente, realizou-se um ensaio microscópico eletrônico de varredura. Os resultados encontrados foram utilizados para escolher qual material seria mais adequado para resistir aos esforços aplicados.

As mesmas amostras passaram pelo preparo para ensaio metalográfico, conforme a norma ASTM E 407: Embutimento em baquelite com a Embutidora Metalográfica EM30D, como pode ser visto o processo nas figuras 3(a), (b), (c) e (d). Em seguida, foi aplicado lixamento, utilizando a Politriz Metalográfica DP-9a e lixas d'água com granulações de \#80, \#160, \#280, \#360, \#400, \#600, \#1200 e \#1500.

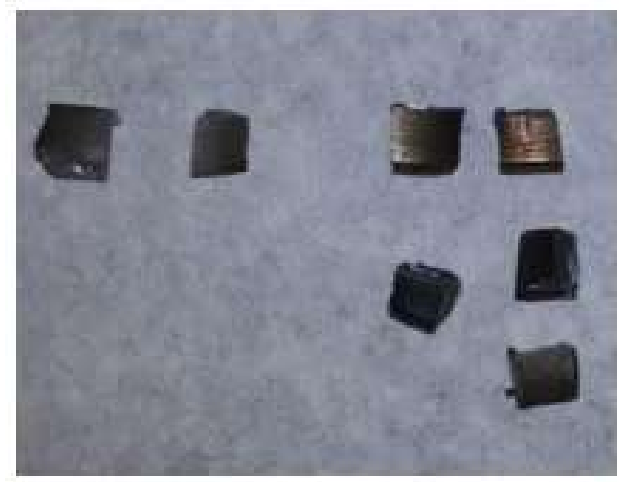

Figura 3a. Amostras a serem analisadas.

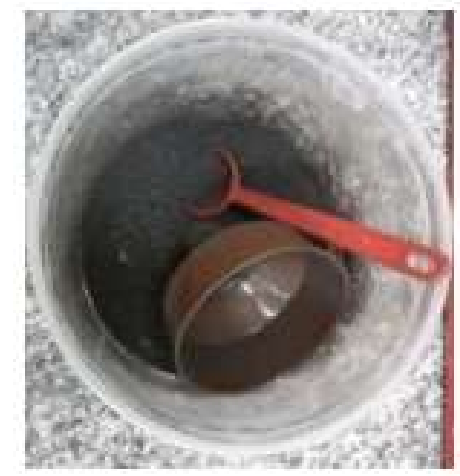

Figura 3b. Material para butimento em baquelite.

IX COEN - Congresso de Engenharias da UFSJ

Interconexão. 


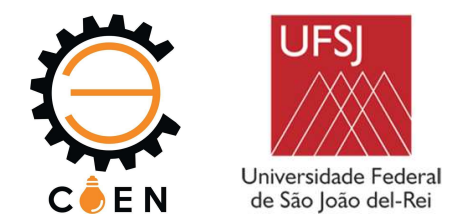

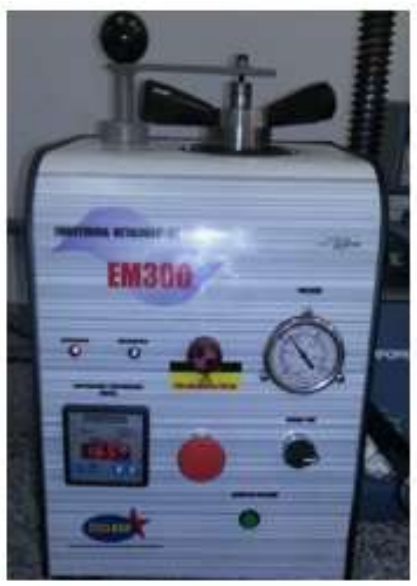

Figura 3c. Máquina embutidora utilizada.

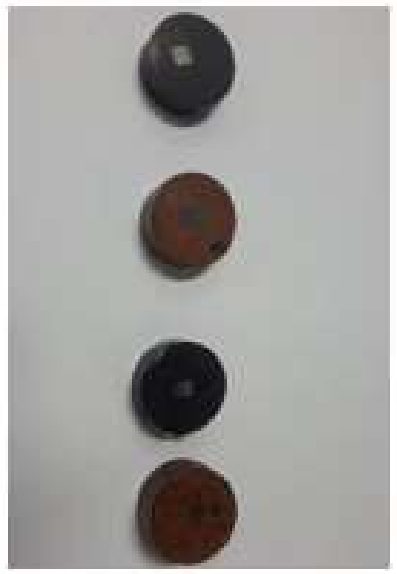

Figura 3d. Amostras preparadas.

Para a micrografia foi feito o polimento com alumina, a fim de obter uma superfície espelhada, ideal para melhor visualização. Em seguida fez-se, separadamente, o ataque químico das amostras, com o ácido Nital. Posteriormente, as amostras foram secas com o intuito de protegê-las. Esse procedimento foi necessário para a revelação da microestrutura do material analisado.

Para a visualização e análise da microestrutura das amostras foi utilizado o Microscópio Metalográfico Olympus BX51 ligado ao computador HP, intel core duo, equipado com o software Analysis gett e a utilização do MEV da marca Hitachi, modelo TM 3000 para aumento das imagens em 50x e 100x, em conjunto com o programa Quantax 70, que permite ver a composição do material analisado.

\section{RESULTADOS}

A dureza da gaiola original foi feita em 5 pontos ao longo da amostra e seu resultado está demonstrado na Tabela 1.

Tabela 1. Dureza da gaiola original

\begin{tabular}{cc}
\hline Pontos & Dureza (HRC) \\
\hline 1 & 59 \\
2 & 58 \\
3 & 58 \\
4 & 59 \\
5 & 59 \\
\hline
\end{tabular}

Com os resultados dos ensaios na gaiola original foi possível observar que o material mais adequado para a fabricação da gaiola homocinética é o aço SAE 8640, por possuir médio teor de carbono, boa resistência mecânica e tenacidade.

A partir disso, foram usinados três componentes para amostras, com o intuito de verificar qual seria mais adequado para a fabricação do novo componente: a $1^{a}$ amostra foi feita do material SAE 8640 com suas propriedades puras; a $2^{\mathrm{a}}$ foi com SAE 8640 e tratamento térmico de têmpera à $850^{\circ} \mathrm{C}$; 
Seja seu próprio

diferencial

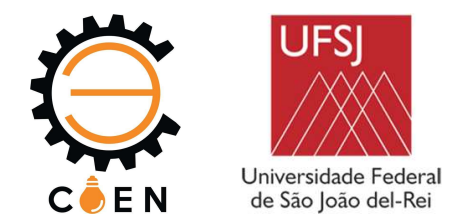

e a $3^{\text {a }}$ foi feita com o SAE 8640 com os tratamentos térmicos de têmpera e revenimento à $850^{\circ} \mathrm{C}$ e $280^{\circ}$ respectivamente.

As imagens das amostras aumentadas em 100x, obtidas no microscópio metalográfico, podem ser vistas nas Figuras 4, 5 e 6.

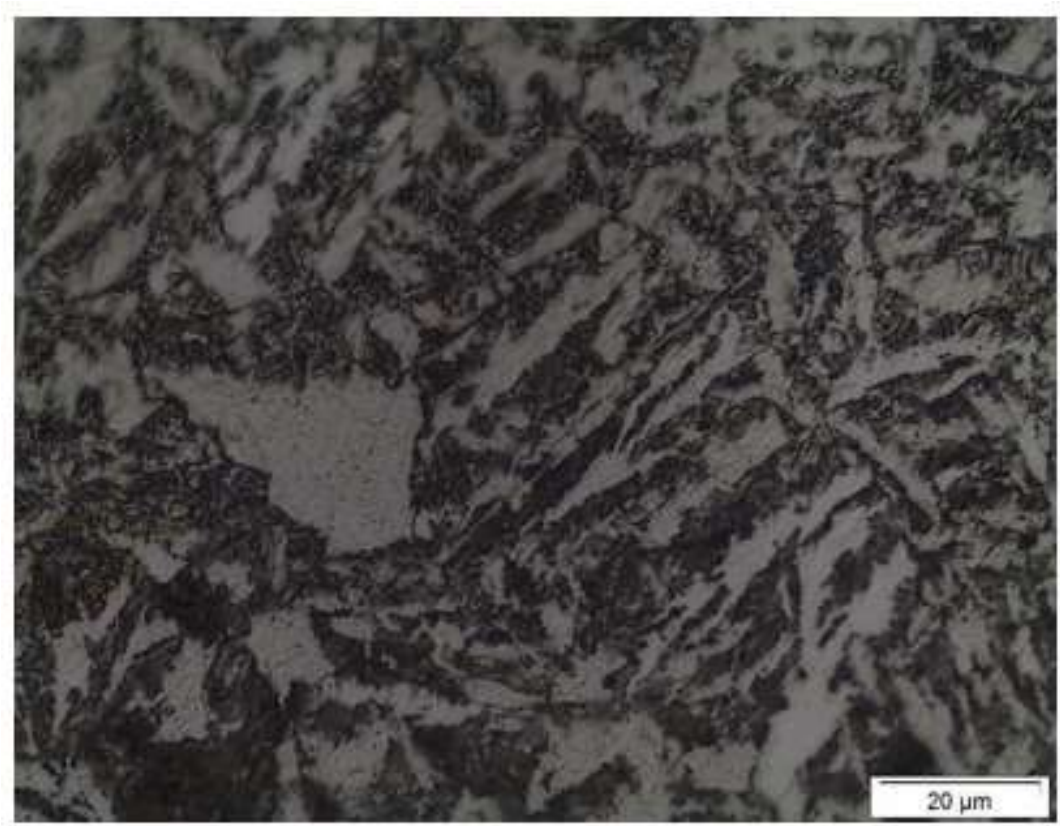

Figura 4. Aço 8640, amostra base (grãos de ferrita (a) - mais claros e cementita - mais escuro), ampliação de 100x.

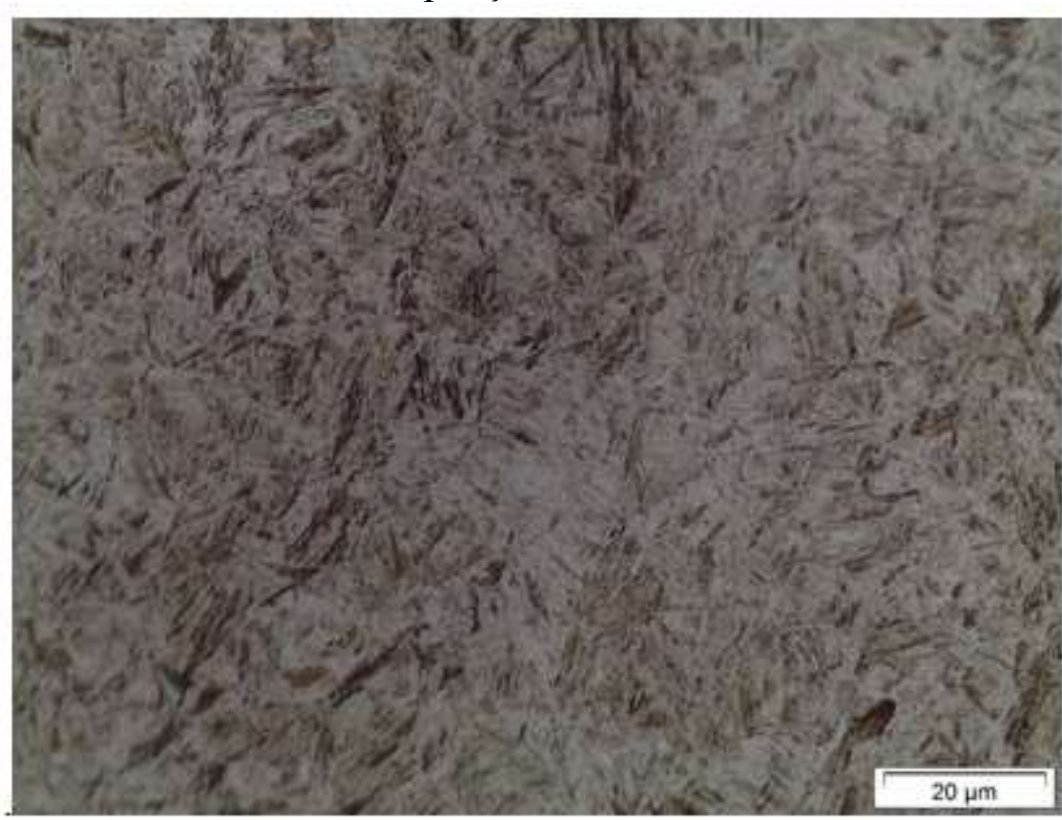

Figura 5. Aço 8640 - Temperado à $850^{\circ} \mathrm{C}$ durante 30 minutos: resfriamento ao óleo, ampliação de 100x.

IX COEN - Congresso de Engenharias da UFSJ Interconexão. 


\section{Seja seu próprio aiferencial}

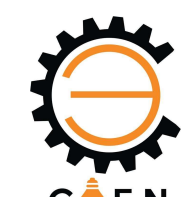

C E N

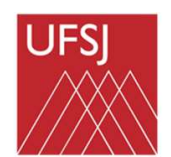

Universidade Federal de São João del-Rei

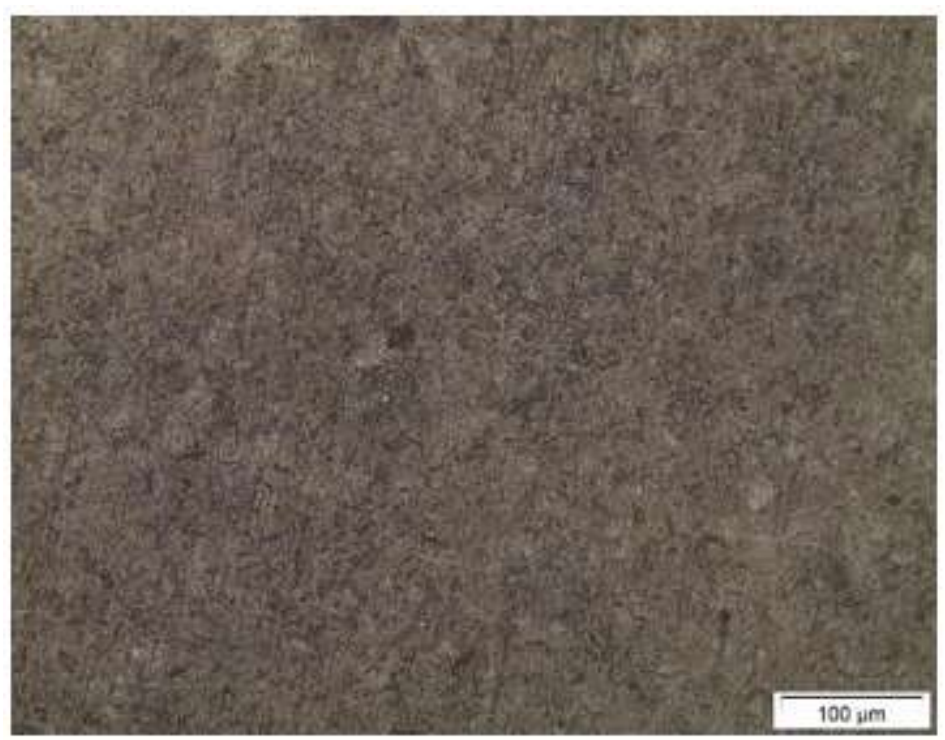

Figura 6. Aço 8640 - Temperado à $850^{\circ} \mathrm{C}$ durante 30 minutos: resfriamento ao óleo e revenimento à $280^{\circ} \mathrm{C}$ durantes 2 horas, ampliação de $50 \mathrm{x}$.

Foi possível observar que a amostra em que houve o tratamento térmico de têmpera obteve um grande resultado de grãos bem definidos de martensita. Já a amostra com tratamento de têmpera e revenimento apresentou uma microestrutura bastante homogênea mostrando regiões com martensita revenida.

Devido ao alto trabalho de atrito e desgaste em que a gaiola homocinética é submetida, optamos por utilizar o componente tratado pelos tratamentos térmicos de têmpera e revenimento, visto que o revenimento é utilizado para corrigir as tensões internas e a dureza excessiva, conseguindo um aumento de tenacidade no componente.

Com a foto retirada no MEV e a análise feita no programa Quantax 70, foi possível fazer uma comparação entre o material do componente original e o aço SAE 8640 temperado e revenido, como pode ser visto na Figura 7 e na Tabela 2.

Tabela 2. Comparação entre as composições químicas do componente original e do componente

\begin{tabular}{c|c|c} 
Elemento & Gaiola Original (\%) & Gaiola Escolhida (\%) \\
\hline $\mathrm{Fe}$ & 56,3 & 52,3 \\
\hline $\mathrm{C}$ & 42,0 & 42,6 \\
\hline $\mathrm{Mn}$ & 0,42 & 0,54 \\
\hline $\mathrm{Ni}$ & 0,28 & 0,35 \\
\hline $\mathrm{Cr}$ & 0,28 & 0,91 \\
\hline $\mathrm{Si}$ & 0,36 & 1,51 \\
\hline $\mathrm{Al}$ & 0,27 & 1,52 \\
\hline $\mathrm{S}$ & 0,08 & 0,36
\end{tabular}

IX COEN - Congresso de Engenharias da UFSJ Interconexão. 


\section{CONCLUSÕES}

A partir dessa comparação, concluiu-se que não houve grandes alterações entre o material original e o escolhido. Isso reforça a necessidade da utilização da engenharia reversa para obtenção da geometria certa da gaiola homocinética, em conjunto com a análise feita do material da peça original, para que se consiga fabricar um novo componente, o mais fiel possível, que resista aos impactos durante o trabalho, sem perdas significativas das características da gaiola original.

Este estudo foi realizado apenas para a gaiola da homocinética, por ser um componente que estava apresentando falhas e não tinha suas dimensões conhecidas pela equipe.

\section{DIREITOS AUTORAIS}

Os autores são os únicos responsáveis pelo conteúdo das informações contidas neste artigo.

\section{REFERÊNCIAS}

HAUTSCH, Oliver. O que é engenharia reversa?. 2009. Disponível em: <https: //www.tecmundo.com.br/pirataria/2808-o-que-e-engenharia-reversa-.htm>. Acesso em: 24 de maio de 2018.

LEITE, M. C., et al. Ensaio de tração mecânica e análise metalográfica do aço SAE 1045. Revista Engenharia em Ação UniToledo, Araçatuba - SP, v. 02, n. 01, p. 83-93, jan/ago. 2017.

PILLON, M. M. Aplicações da Engenharia Reversa no Desenvolvimento de Produtos. 2015. 66f. Trabalho de Conclusão de Curso - Centro Universitário Eurípedes de Marília - UNIVEM. Marília, SP, 2015.

PONTICELLI, C., SUSKI,C.A. O avanço do desenvolvimento de produtos através da engenharia reversa. Revista da UNIFEBE, 2017 - periodicos.unifebe.edu.br, v. 1, n. 8, jan/jul. 2017.

SANTOS dos, E. P. CAD/CAM/Usinagem CNC integrado a engenharia reversa. 2017. 57 p. Dissertação (Mestrado em Engenharia Mecânica)- Faculdade de Engenharia, UNESP, Ilha Solteira- SP, 2017.

\section{REVERSE ENGINEERING METHOD FOR THE DIMENSIONING OF HOMOKINECT CAGE}

Igor Damasceno Baeta (igor.d.mec3@gmail.com), Marcelo Esposte Purgato (marceloesposte@gmail.com), Alexandre Chaves de Melo (alexandrechavesdemelo@gmail.com),

Marina Paiva Rodrigues (mapaivarodrigues@yahoo.com.br)

Universidade Federal de São João Del Rei (UFSJ), Curso de Engenharia Mecânica, Praça Frei Orlando-170, Centro, 36307-904, São João Del Rei, Minas Gerais, Brasil.

Universidade Federal de São João Del Rei (UFSJ), Departamento de Engenharia Mecânica (DEMEC), Praça Frei Orlando-170, Centro,36307-904, (32)3379-5879, São João Del Rei, Minas Gerais, Brasil.

ABSTRACT: The prototype Baja is a off road vehicle produced for competitions. In those competitions this prototype is subjected to tests of several modalities. The subsistem of powertrain is responsable for the transmission of the torque and power produced by the engine to the wheels. The homokinects' cage is a part of the homokinect joint, component is directly coupled in the wheel hub. The present article will deal the use of reverse engineering to produce new homokinects' cage, analyzing the property of the materials according to the component's requirements. This analyze was motivated by recurrent cage's breaks, and the objective of this is to produce a new cage more resistant than the original one, analyzing yours structural properties.

KEYWORDS: Homokinect, baja, homokinect cage 